\title{
LA AUTOGESTIÓN DE USUARIOS HIDROAGRÍCOLAS DEL VALLE DE MEXICALI. EFECTOS DEL PROCESO DE TRANSFERENCIA ${ }^{1}$
}

\section{$\longrightarrow$ \\ RESU M E N}

En este trabajo se presentan los resultados parciales de investigación sobre las implicaciones agroproductivas y ambientales de la política de modernización del campo en el valle de Mexicali. La vertiente analizada, arroja que el proceso de transferencia del distrito de riego a los usuarios tomó distintos significados en los diferentes periodos de inicio, estabilización y consolidación después de cinco años de su formalización. Contraria a la postura inicial, en la actualidad los usuarios consideran quefue benéfico y quetiendea desarrollar su capacidad deautogestión, sobre todo para operar el agua y la infraestructura. No obstante, se encuentra que aún existen diversas limitaciones deíndoleorganizacional principalmente, referidas a capacitación gerencial de los usuarios, coordinación interna e institucional, desarrollo organizativo y normatividad, aspectos que resultan determinantes del lento avanceen el logro de los objetivos centrales dela transferencia: autosuficiencia y uso eficiente del agua.

\section{A BSTRACT}

The present work exhibits the partial research results on the agriproductive and environmental implications of thefarmland modernization policy that was applied in the Mexicali Valley. The angle that is analyzed in this document shows that the transfer process of theirrigation districts to the users took various meanings during the different initiation, stabilization and consolidations periods after five years of its formalization. Unlike the initial position, at present the users find that it was beneficial, and that it tends to develep their capabilities for selfprocedures, especially with regards to theoperation of water and infrastructure. However, thereareseveral constraints still, mainly dealing with organizational issues that refer to the management training of users, internal and institutional coordination, organizational development and normativeness. Such aspects arekey factors for theslow progress in accomplishing the main objectives of the transfer: self-sufficiency and efficient water use.

*Director de la Dirección Regional Mexicali de El Colegio de la Frotera norte. Correo electrónico: acortez@telnor.net

${ }^{1}$ Resultados parciales del estudio "La modernización del campo: implicaciones agroproductivas y ambientales en el valle de Mexicali", El Colegio dela Frontera N orte, Dirección Regional Mexicali-Fundación Colef, 1997. 


\section{INTRODUCCIÓN}

En el contexto dela descentralización defunciones del sector hidráulico a través de la transferencia de los distritos de riego en 1993, las asociaciones deusuarios (AU) delos módul os deriego seestablecieron en la primera fase de transferencia, como las nuevas instancias operadoras, asumiendo la responsabilidad para participar en la formulación y ejecución de programas y acciones para mejorar el manejo y distribución del agua, el desarrollo de infraestructura hidráulica y de conservación del recurso hídrico. Sin embargo, estas instancias han enfrentado, desde entonces, limitaciones para llevar a cabo una autogestión eficienteen busca dela maximización debeneficios sociales, técnicos y económicos. Esto, en principio seasocia a la incipientepero creciente experiencia, el bajo nivel decapacitación directivo-gerencial y el lento desarrollo organizacional para administrar y operar las áreas deriego.

La normatividad básica de las mencionadas asociaciones es la Ley federal de aguas, actualizada en la Ley de aguas nacionales (LAN) de 1992, de donde se deriva el Instructivo de operación, conservación y administración (IOCA) para la elaboración delos estatutos delas AU, en los queseestableceel objeto desu formación, así como lo relativo alos derechos y obligaciones de los asociados, las cuotas y actividades inherentes a la asamblea, misma que se constituye como el máximo órgano deautoridad interno.

En este sentido, la autogestión se explicita en la reglamentación y se refierea lo siguiente: a) participar en la administración, operación, conservación y mejoramiento de las obras de infraestructura hidroagrícola; b) ofrecer servicio deriego a los asociados; c) recaudar y administrar cuotas; d) complementar las obras de infraestructura; e) realizar obras de conservación; f) adquirir bienes muebles e inmuebles, y g) capacitar a losasociados y empleadosen laoptimización del uso de los recursos hidráulicos.

El cumplimiento delos derechos y obligaciones de los asociadoses un parámetro adicional dela autogestión. Los derechos del usuario se concretan en recibir el servicio de riego y los volúmenes deagua en la calidad, cantidad - de acuerdo con la dotación preestablecida- y 
oportunidad requeridas para llevar a cabo sus labores productivas. Para ello, deben efectuar el pago decuotas, entregar sus programas de siembra, presentar requerimientos volumétricos de agua de acuerdo con el cultivo a establecer, evitar desperdicios de agua, así como construir y mantener obras menores de riego.

Con el anterior enfoque dereciprocidad, setieneuna evaluación de autogestión más clara apoyada en indicadores técnico-normativos y económico-productivos. ${ }^{2}$

\section{Metodología}

Lainformación seobtuvo a través dela utilización dedosinstrumentos: las entrevistas a profundidad que se aplicaron a informantes clave y un cuestionario para recabar información diversa.

\section{DISEÑO DE INSTRUMENTOS}

1. Se elaboró un cuestionario de preguntas cerradas para obtener información rel evanterelacionada con las vertientes planteadas. Los temas que integran el cuestionario son:

- Actitudes y acciones de los usuarios respecto a la transferencia del distrito deriego.

- Autogestión derecursos hidroagrícolas.

- Eficiencia en el uso y manejo del agua.

\footnotetext{
${ }^{2}$ Los indicadores se basan en dos aspectos: 1) análisis de la normatividad y de las funciones de operación, conservación y administración, y 2) análisis de la organización, representatividad y formas de participación de los usuarios. Estos puntos se apoyan en el trabajo de León Duarte (1996). “Diagnóstico dela situación del distrito de riego 051, costa de Hermosillo ante los cambios en la gestión del agua". A simismo, tanto en el presente trabajo como en el que se cita, se considera quela entidad deautogestión del agua, en este caso el módulo de riego, realiza una actividad degestión deconflictos entreusuarios quecompiten por el mismo recurso y que a menudo desconocen cómo interactúan, lo que los lleva a perjudicarse, o en su caso, beneficiarse de manera recíproca.
} 
2. Se elaboró una guía temática para las entrevistas a profundidad, con el fin de obtener información cualitativa que permita un análisis integral de cada una delas vertientes de estudio.

\section{DISEÑOS DE MUESTRA}

Desde las perspectivas de uso y manejo de recursos hidráulicos comprendidos en las dos primeras vertientes de modernización analizadas, el módulo de riego reúnela característica de ser la unidad deautogestión hidroagrícola. En el marco muestral para este caso, la población incluyea los usuarios registrados en el padrón (único) dela Asociación deUsuarios del Módulo deRiego Núm. 15, margen derecha del Río Colorado, A.C., ${ }^{3}$ constituidaen 1993 por un total de668 usuarios, de los cuales 499 pertenecen al régimen ejidal y 165 son colonos y/ o propietarios privados. La superficie física del área de riego abarca 9803 ha. En esta delimitación territorial se establecen los cultivos representativos de la diversidad agrícola del valle de Mexicali, pero también de la tipología de productores y condiciones físicas como los tipos desuelosy sistema deriego. Deestemarco seobtuvo una muestra aleatoria de conglomerados, de tal manera que se consideraran y agruparan las variables de interés para el estudio.

\section{CARACTERÍSTICAS DE LA ZONA DE ESTUDIO}

El distrito de riego 014, Río Colorado, se ubica en la planicie costera aluvial correspondiente a la parte terminal de la cuenca baja del Río Colorado, y abarca los valles agrícolas de M exicali, Baja California y San Luis Río Colorado, Sonora. La altitud dela región fluctúa entrelos -2 hasta los $45 \mathrm{~m}$ sobre el nivel del mar.

${ }^{3}$ Cabeseñalar que, por las características del proceso mismo detransferencia, al gunos usuarios trabajan en tierras circunscritas a módulos vecinos $(16,18,19)$ fuera del módulo donde están registrados (15). 
En el distrito se reportan oficialmente 250000 ha dominadas con obras hidráulicas y una superficie regable de 207965 ha distribuidas en subciclos agrícolas de la siguiente manera: 59\% en otoño-invierno, $26 \%$ en primavera-verano, y el $15 \%$ restante para cultivos perennes.

El módulo deriego núm. 15, margen derecha del Río Colorado, se encuentra ubicado en la zona nortedel valle deM exicali y colindacon el sureste de la zona urbana. En esta subregión, la superficie ejidal predomina con 8016 ha y 510 usuarios, sobre 1912 ha y 168 usuarios dela pequeña propiedad. Por rangos dedotación detierra, del padrón de usuarios se desprende que $26 \%$ cuenta con hasta 10 ha, $73 \%$ entre 11 y 20 ha, y $1 \%$ están dotados con más de 20 ha. En cuanto a tipos de suelo, los ligeros cubren $8 \%$, los medios $22 \%$, y los pesados $70 \%$ dela superficie del módulo (ver cuadro 1), (Cortez, 1996).

CUADRO 1. Plan de riegos normal del módulo de riego núm. 15.

\begin{tabular}{|c|c|l|c|}
\hline Cultivo & Superficie(ha) & Mes & Volumen $\left(\mathrm{Mm}^{3}\right)$ \\
\hline Otoño-invierno & 4900 & Octubre & 3703 \\
Trigo & 2700 & Noviembre & 6860 \\
Rye-Grass & 1650 & Diciembre & 8519 \\
Cártamo & 100 & Enero & 8673 \\
Varios & 450 & Febrero & 10298 \\
Perennes & 1500 & Marzo & 14029 \\
Alfalfa & 1500 & Abril & 12936 \\
Primavera-verano & 2800 & Mayo & 12245 \\
Algodonero & 1000 & Junio & 11319 \\
Sorgo forrajero & 1440 & Julio & 8961 \\
Sorgo grano & 110 & Agosto & 4659 \\
Varios & 250 & Septiembre & 3334 \\
Total anual & 9150 & Total anual & 105543 \\
\hline
\end{tabular}

FuentE: Elaboración propia a partir de datos del módulo 15. 
En estetrabajo seentiendea la autogestión derecursos hidroagrícolas, como toda actividad sustantiva realizada por los usuarios directos de los mismos relacionada con la administración, operación y conservación del agua de riego y desarrollo de infraestructura, contemplando en éstas los vínculos con otros usuarios, entidades normativasy operativas de los tres niveles de gobierno y entes sociales de la ciudad. En estas actividades, los socios participan desdediferentes posiciones: dirección, gerencia y receptor del servicio de riego. Los dos primeros, avalados por la asamblea general, deciden, ejecutan y establecen los programas y acciones determinadas en beneficio de los últimos. Así pues, los usuarios manifiestan, en su actividad deautogestión, una posición dual y dinámica, misma quese adecúa a los cambios periódicosocasionados por las al ternancias administrativas tipificadas en la reglamentación.

\section{FUNDAMENTOS DE AUTOGESTIÓN EN LA AU DEL MÓDULO DE RIEGO}

El tema de gestión de este recurso, tanto a nivel nacional como de cuencas, es bien acogido por muchas personas e instituciones, sin embargo, aún no logra plasmarse en sistemas sólidos y estables, salvo donde se han realizado grandes inversiones en obras hidráulicas (Dourojeanni, 1994). Con este antecedente global, resulta prioritario revisar al gunos aspectos, particularmente en los distritos de riego en México después de efectuada la transferencia a los usuarios y donde las AU formadas juegan un papel protagónico.

En el valle de Mexicali, la autogestión del agua por parte de los usuarios agrícolas muestra limitantes, muchas de ellas originadas en la propia formación delas AU einicio defunciones para llevar a cabo la operación dela red hidráulica menor, la administración delos módulos deriego y la conservación y desarrollo de infraestructura. La “vertiginosidad" dela implantación delas Au, la mínima capacitación técnica y administrativa de los usuarios para recibir tal responsabilidad, y las malas condiciones de la infraestructura que la Comisión Nacional del Agua (CNA) entregóa los usuarios, entreotros, fueron y son aún factores 
determinantes delas deficiencias. En términosgenerales, a estosnuevos entes del manejo del agua se les dieron amplias facultades, ya que debían cumplir con requisitos tales como: ser económicamente eficientes, autosuficientes y competitivos; tener orientación social para favorecer la equidad, y permitir la participación social, entre otras. Lo anterior no ha representado una tarea sencilla. Desdela transferencia del distrito deriego, seobserva que paulatinamenteseha ido desarroIlando la capacidad para atender los aspectos sustantivos, lo que les permite cumplir con algunas funciones básicas, como las de evitar, reducir, y en su caso, solucionar conflictos entre usuarios del agua y la infraestructura.

\section{LA BASE NORMATIVA}

Los documentos básicos donde la AU soporta sus actividades de autogestión son el IOCA, mismo quesefundamenta en los "A nexos del Título de Concesión" (Comisión Nacional del Agua, 1993). Este documento contiene información específica sobre la normatividad, geografía y aspectos técnicos dela región deinfluencia del módulo de riego, y se compone de los siguientes apartados: a) plano general del distrito y del módulo de riego; b) inventario de infraestructura hidroagrícola; c) padrón deusuarios; d) actaconstitutiva dela asociación civil, incluyendo los estatutos que rigen, en primera instancia la organización, y en segunda, delinean el esquema de autogestión. AI respecto, se muestra un rezago significativo en la mayoría de los componentes de dicho documento básico, ya queel mismo se elaboró para efectos de la transferencia, y hasta ahora no ha sido actual izado, considerando las adecuaciones necesarias para cada caso espeć́fico, es decir, para cada una de las AU formadas.

${ }^{4}$ El documento "A nexos del Título de Concesión" se elaboró por la Comisión Nacional del Agua de manera similar para las 23 AU formadas en el Distrito de riego. 
El padrón deusuarios y los instrumentos reglamentarios particulares muestran deficiencias significativas, pues el no contar con un documento veraz que identifiquecual itativa y cuantitativamentealos integrantes de la asociación - personas que hacen uso de los recursos hidráulicos-, deentradainducediversos desajustes en las actividades normales del módulo deriego, mismosquesetraducen en ineficiencias decarácter técnico-administrativo y operativo. La falta de precisión en el conocimiento delas condiciones deusufructo, tanto dela superficie de labor como de volúmenes de agua de riego, representa un factor limitativo elemental para realizar una autogestión eficiente.

Al respecto, la revisión inicial dela información basada en el padrón de usuarios, arrojó que sólo 30\% de la superficie de riego se trabajó directamente por el usuario propietario, mientras que el resto de la proporción desuperficiedel módulo seencontraba en arrendamiento. Esto implica queusuarios del módulo, además deexplotar su derecho normal deriego, ${ }^{5}$ laboran una superficieadicional deextensión variable que oscila en el rango de 40 a 1500 ha, atendidas en la modalidad individual, en asociación familiar $u$ otro tipo. Cabe señalar que esta información no se encontró en los registros oficiales, no obstante que las repercusiones de dichas irregularidades en los registros de superficie por individuo derechoso, representan un problema básico para realizar demanera eficientelas actividades sustantivas, sobre todo para la programación anual devolúmenes, cobro y seguimiento deprocesos como el servicio deriego.

Los estatutos de la AU elaborados como requisito para la transferencia, muestran una gran cantidad devacíos. El documento establece puntos fundamentales tales como los objetivos de la asociación, derechos y obligaciones de los asociados, y en diversos capítulos los aspectos de operación, administración y conservación del agua e

${ }^{5}$ El promedio regional por usuario es de 20 ha de riego, que en términos de lámina de agua o volumen es de $12000 \mathrm{~m}^{3} /$ ha que se obtiene ponderando la dotación basada en el tipo de cultivo y la eficiencia de distribución y riego ( $50 \%$ general). Este volumen es el multiplicador dela superficie adicional que se labore. 
infraestructura. Sin embargo, en términos decontenido y aplicación se encuentran deficiencias, ya que si bien se plasman los aspectos gené ricos, no seal canza a comprender especificidades decada AU. El documento seelaboró partiendo dela normatividad general, y en la actualidad no se ha revisado ni adaptado a cada subregión y condición particular de autogestión; en este caso, la del módulo de riego número 15.

Adicionalmente, se detectan dificultades para lograr el franco cumplimiento de los estatutos en al gunos aspectos, y en otros casos los encargados de la administración (autoridades de la AU) se ven limitados en sus facultades para ejercer acciones. Esto se denota particularmente en los casos de ajuste de cuotas por servicio de riego, contratación de financiamientos y aplicación de sanciones; los dos primeros puntos se encuentran fuera del al cance del nivel individual de autogestión de la AU, puesto que obedecen a niveles de decisión superior (comitéhidráulico), ${ }^{6}$ en tanto queel tercer aspecto, haresultado un reto difícil de lograr para las autoridades de la AU, debido a lo complicado que es aplicar el reglamento de manera estricta para sancionar, cuando la cultura inducida por el organismo oficial antes dela transferencia no lo consideró. Sin embargo, éstees un punto clave que paulatinamente se ha ido contemplando en la autogestión con el objeto delograr eficazmentelas funciones sustantivas, particularmente la de eficientar el uso del agua.

Lo anterior permite distinguir la necesidad de adecuar los reglamentos detal manera queselogren atender condiciones específicas de cada subregión de riego, ampliando aspectos de tipificación de mecanismos decumplimiento deobligaciones por partedelos usuarios, deaplicación decuotas y derelaciones interinstitucionales con los tres niveles degobierno y lasociedad quecompitepor el recurso hidráulico.

${ }^{6}$ Con base en el artículo 66 de la LAN, en el distrito de riego se establece el comité hidráulico para actuar como órgano colegiado de concertación para el manejo del agua e infraestructura, y es la instancia donde los usuarios toman decisiones y acuerdan acciones necesarias para una adecuada administración del distrito. 
Ésta es una actividad quecompeteal comitéhidráulico, y querepresenta el reto para mostrar su autonomía técnica y de gestión para atender la problemática relacionada con los recursos hidráulicos.

\section{CONTROL ESTADÍSTICO DE INFORMACIÓN}

El nivel desistematización de la información, que considera la forma de registro, clasificación y cuantificación de la estadística generada, tanto en el ámbito directivo como técnico del módulo de riego, es un factor adicional indicador dela capacidad de autogestión.

La revisión de los controles internos de la Au muestra que en la requisitación y operatividad dela gestión hidroagrícola seutilizan un total de 43 formatos (13 referentes a la actividad de operación, 19 para conservación y 11 deadministración) de captura y organización dela amplia gama de información de uso interno en apoyo a la toma de decisiones de corto, mediano y largo plazo.

En el cuadro 2 se distingue el grado mínimo de automatización, sistematización e integración de los controles estadísticos, pese a la relación quemantienen las tres actividades primordiales en laactividad del módulo deriego. Sedeterminó queentre $75 \%$ y $80 \%$ delos formatos son capturados manualmente y contemplan la información sobre conservación y operación. La información del ámbito administrativo en términos generales se captura y registra de manera informatizada.

Por otra parte, es evidente la influencia de la entidad normativa oficial en el diseño de instrumentos de control, pues, a excepción de los formatos para actividades administrativas, los de captura son similares a los queseutilizaron por aquella entidad durantesu gestión previa a la transferencia.

De ello se deduce que, independientemente de la utilidad práctica y funcional que puede ofrecer el uso de los esquemas mixtos (sobre todo en la primera fase de la transferencia del distrito), representa un factor inhibidor de la iniciativa de los usuarios en la búsqueda de distintos esquemas y sistemas de organización dela información, o la modificación, en su caso, de los ya existentes, en el entendido de que en su momento, aquellos se adaptaron a condiciones y situaciones prevalecientes. 


\section{CUADRO 2. Resumen de la base documental y formatos utilizados en la autogestión.}

\begin{tabular}{|c|c|c|}
\hline Operación & Conservación & Administración \\
\hline $\begin{array}{l}\text { 1. Avancedesiembrasprogramado } \\
\text { y realizado (ha/ cultivo/ mes) }\end{array}$ & $\begin{array}{l}\text { 1. Trabajos realizados por } \\
\text { cuadrilla, concentrado } \\
\text { 2. Mantenimiento decompuer- } \\
\text { tasy mecanismos }\end{array}$ & $\begin{array}{l}\text { 1. Recibos depago por servicio } \\
\text { deriego (padrón chico) }\end{array}$ \\
\hline $\begin{array}{l}\text { 2. Eficiencias diarias } \\
\text { (cultivo/ díariego) }\end{array}$ & $\begin{array}{l}\text { 3. Informediario detrabajo } \\
\text { demaquinaria }\end{array}$ & $\begin{array}{l}\text { 2. Solicitud desuperficie } \\
\text { deriego }\end{array}$ \\
\hline $\begin{array}{l}\text { 3. Programa derequerimientos } \\
\text { deagua por año agrícola } \\
\text { (mes/ decena/ volúmenes) }\end{array}$ & $\begin{array}{l}\text { 4. Costo deoperación y mante- } \\
\text { nimiento demaquinaria }\end{array}$ & $\begin{array}{l}\text { 3. Solicitud detransferencia } \\
\text { provisional dederecho de } \\
\text { riego }\end{array}$ \\
\hline 4. Registro diario deriegos(D-6) & 5. Avancedemaquinaria & $\begin{array}{l}\text { 4. Listas deasistencia de } \\
\text { asambleadeusuarios }\end{array}$ \\
\hline $\begin{array}{l}\text { 5. Manejoy distribución del agua } \\
\text { programada-recibida } \\
\text { (mes/ decena/ volúmenes) }\end{array}$ & $\begin{array}{l}\text { 6. Trabajo deconservación } \\
\text { normal }\end{array}$ & 5. Reportedecampo \\
\hline $\begin{array}{l}\text { 6. Eficiencia por seccionesy } \\
\text { zonas (sección/ gasto/ eficiencia) }\end{array}$ & \begin{tabular}{|l|} 
7.Informedereparacióny \\
mantenimiento del revesti- \\
miento decanales y estructuras
\end{tabular} & $\begin{array}{l}\text { 6. Intervalosy seguimientos } \\
\text { delos riegos diarios repor- } \\
\text { tados por jefes desección }\end{array}$ \\
\hline $\begin{array}{l}\text { 7. Solicitud deservicio de } \\
\text { riego (usuarios) }\end{array}$ & $\begin{array}{l}\text { 8. Mantenimiento en deshierbe } \\
\text { y limpiaamano }\end{array}$ & 7. Facturapor servicio deriego \\
\hline $\begin{array}{l}\text { 8. Control deservicio deriego } \\
\text { (depósito\$/ litros) }\end{array}$ & $\begin{array}{l}\text { 9. Tablaresumen derelación } \\
\text { demateriales por cuadrilla }\end{array}$ & $\begin{array}{l}\text { 8. Poliza dediario } \\
\text { 9. Poliza deingresos } \\
\text { 10. Polizadecheque }\end{array}$ \\
\hline $\begin{array}{l}\text { 9. Reportedepreparación de } \\
\text { tierras (resumen DR 014) }\end{array}$ & 10. Avancemensual deobras & 11. Contratolaboral \\
\hline $\begin{array}{l}\text { 10. Reportediario dedistribución } \\
\text { deagua (usuario/ localizac// culti- } \\
\text { vo/ aguaentregada/ superficie) }\end{array}$ & $\begin{array}{l}\text { 11. Gastosmensuales dela } \\
\text { cuadrilladereparaciones } \\
\text { 12. Gastosmensuales dela } \\
\text { cuadrilladesoldadores }\end{array}$ & $\begin{array}{l}\text { 12. Actividades realizadas } \\
\text { por los directivos }\end{array}$ \\
\hline $\begin{array}{l}\text { 11. Reportedepreparación de } \\
\text { tierras por subcido }\end{array}$ & $\begin{array}{l}\text { 13. Memoriadescriptivade } \\
\text { trabajos deconservación }\end{array}$ & \\
\hline 12. Avancediario deriegos (D-16) & 14. Orden detrabajo & \\
\hline \multirow[t]{4}{*}{ 13. Demanda diariadeagua(D-10) } & 15. Tabladedesazolvepor tramos & \\
\hline & 16. Tabla decorte & \\
\hline & $\begin{array}{l}\text { 17. Trabajos deconservaciónaes- } \\
\text { tructurasen red dedistribución }\end{array}$ & \\
\hline & $\begin{array}{l}\text { 18. Informedeoperación de } \\
\text { maquinaria }\end{array}$ & \\
\hline
\end{tabular}

FuENTE: Elaboración propia con base en información del módulo de riego número 15, distrito de riego 014, Río Colorado. 
Con el afán de adecuar a las condiciones actuales y profundizar en el tipo de información y datos requeridos para apoyar la toma de decisiones, en el contexto deautogestión sehacenecesario independizar el diseño de formatos de organización y manejo de la información, siempre dentro del marco normativo que la CNA debevigilar.

TRANSFERENCIA Y AUTOGESTIÓN DE USUARIOS

\section{LA PERCEPCIÓN DE USUARIOS SOBRE EL PROCESO DE TRANSFERENCIA}

El análisis dela autogestión de usuarios partederevisar su percepción sobre el proceso de transferencia, con objeto de diferenciar el cambio de posturas en escenarios comparativos de tiempo. ${ }^{7}$

La postura se indicó considerando las siguientes respuestas: a) a favor, b) en contra, c) indiferentesy d) dudosa. Losanálisis estadísticos decontingencia arrojan diferencias significativas respecto a la postura de los usuarios antes versus después de la transferencia.

Así, sobresale el cambio positivo de la opción a favor, cuya proporción pasa de $43 \%$ a $67 \%$ deun periodo a otro. El comportamiento delas demás opciones es en sentido opuesto. En el periodo inicial con postura en contra, indiferente y dudosa, se encontraba $57 \%$ de los usuarios, lo queevidenciala inconsistenciainicial del proceso, quesin duda dificultó su avance. Sin embargo, los resultados muestran que en la percepción general del proceso detransferencia, los usuarios han incrementado, demanera paulatina, su confianza en proporción inversa aladisminución deposturasnegativas, principalmenteladeindiferente (ver cuadro 3).

${ }^{7}$ El estatus de tiempo anterior a la transferencia del distrito de riego a los usuarios (antes), contempla el periodo inmediato al proceso, y pudiera ubicarse hasta antes de 1993. El estatus posterior (después) considera el tiempo actual quepuntualmente abarca al ciclo agrícola 1997. 
Cuadro 3. Postura de usuarios respecto al proceso de transferencia del distrito de riego.

\begin{tabular}{|l|c|c|c|}
\hline Posturadeusuarios & $\begin{array}{c}\text { Proporción de } \\
\text { usuariosantes de } \\
\text { latransferencia } \\
(1992-1993) .\end{array}$ & $\begin{array}{c}\text { Proporción de } \\
\text { usuariosdespués } \\
\text { delatransferencia } \\
(1996-1997) .\end{array}$ & $\begin{array}{c}\text { Tasadecambio } \\
\text { (\%) depostura } \\
\text { deusuarios }\end{array}$ \\
\hline A favor & 43.3 & 66.7 & +23.4 \\
En contra & 30.0 & 21.7 & -7.3 \\
Indiferente & 18.3 & 10.0 & -8.3 \\
Dudosa & 8.3 & 1.7 & -6.6 \\
\hline
\end{tabular}

FuENTE: Elaboración propia con base en resultados de la encuesta "La modernización del campo: implicaciones agroproductivas y ambientales en el valle de Mexicali", sección II, Percepción sobre la transferencia del distrito de riego.

Una mejor explicación del cambio observado en las posturas de los usuarios, se obtiene al considerar algunos el ementos de juicio. Primeramente, la desconfianza manifiesta del primer periodo analizado, se soporta en la forma en que sellevó a cabo el proceso, caracterizado por su vertiginosa aplicación, fal ta de información oportuna, ausencia de estudios de impacto y desinformación intra e intersectores de usuarios e incluso autoridades promotoras del proceso en aquel entonces. El manejo adecuado de las formas podría haber inducido una aceptación inicial sin mayores problemas, disminuido los costos deaplicación y, así, permitir un mejor avance del proceso.

La modificación deactitudes y la identificación de los principales cambios positivosy/ o negativos al transferir el distrito, es un análisis complementario. Se destaca que $62 \%$ de usuarios indican tener una mayor participación en las actividades sustantivas del módulo de riego, y sólo una proporción de $8 \%$ señaló que existe divisionismo (ver cuadro 4). 
CuADRo 4. Actitud actual de los usuarios del módulo de riego ante la transferencia de funciones operativas, administrativas y de conservación del agua einfraestructura.

\begin{tabular}{|l|c|}
\hline Actitud & Proporción deusuarios (\%) \\
\hline Mayor participación & 61.7 \\
Menor participación & 18.3 \\
Indiferente & 10.0 \\
Divisionismo & 8.3 \\
Otra & 1.7 \\
\hline
\end{tabular}

FUENTE: Elaboración propia con base en resultados de la encuesta "La modernización del campo: implicaciones agroproductivas y ambientales en el valle de Mexicali", secciones II y III, Percepción sobre la transferencia del distrito de riego y Gestión de recursos hidroagrícolas.

Los puntos señalados muestran congruencia con el siguiente apartado, donde básicamente se distinguen cambios positivos derivados del proceso de transferencia (sólo una proporción de $27 \%$ delos usuarios dijo haber observado cambios negativos). Los cambios positivos serelacionan, en orden deprioridad, con: 1) disminución de trámites burocráticos referentes al servicio deriego; 2) mayor nivel de inversión para infraestructura hidráulica, y 3) mejoramiento de la eficiencia en el uso y manejo del agua deriego a nivel demódulo. Los dos primeros aspectos en conjunto se mencionan en $64 \%$ de los casos. A simismo, el tercer punto se presenta en $20 \%$ de los casos.

El análisis estadístico muestra un cambio real y con tendencia positiva en la percepción de los usuarios respecto al proceso de transferencia del distrito entre un periodo y otro. Esto coincideen parte con las afirmaciones de Dourojeanni, respecto a los entes de gestión funcionales y las condiciones para ello, donde el mayor nivel de inversión para infraestructura es un factor determinante. 
En la situación local estudiada, la explicación sobreel cambio en la percepción y la nueva actitud participativa de los usuarios se basa en los factoresqueindican la actividad deautogestión y operación técnica delos recursos hidráulicos. Así, seencontró dependencia entreal gunas respuestas quecontemplan tal combinación, a través del análisis dejicuadrada para pruebas deindependencia. Los coeficientes deconfianza para dichas relaciones se anotan en el cuadro 5, donde se observa la dependencia del valor dela variableproporción deusuarios con postura actual (1997) a favor dela transferencia, contrastada con los valores de las proporciones de usuarios que no han observado dificultades en la labor del módulo de riego, con las de usuarios que indicaron que después dela transferencia se ha incrementado la realización deobras

CUADRO 5. Relación de respuestas y coeficientes de confianza ji-cuadrada para la prueba de independencia (eventos dependientes).

\begin{tabular}{|c|c|c|c|c|c|}
\hline $\begin{array}{c}\text { Percepción sobrela } \\
\text { transferenciadel }\end{array}$ & \multicolumn{3}{|c|}{$\begin{array}{l}\text { Autogestión derecursos } \\
\text { hidroagrícolas }\end{array}$} & \multicolumn{2}{|c|}{$\begin{array}{l}\text { Uso y manejo del agua } \\
\text { deriego en el módulo }\end{array}$} \\
\hline $\begin{array}{l}\text { P: ¿Cuál essu postura } \\
\text { actualmente? }\end{array}$ & $\begin{array}{l}\text { P: ¿Haobservado } \\
\text { dificultadesen el } \\
\text { trabajodel } \\
\text { módulo? }\end{array}$ & $\begin{array}{l}\text { P:Sehaincre } \\
\text { mentadola } \\
\text { realizaciónde } \\
\text { obras después } \\
\text { delatransfe } \\
\text { rencia? }\end{array}$ & $\begin{array}{l}\text { P:¿Cómoson } \\
\text { ahoralostrá } \\
\text { mitesparae } \\
\text { servicio de } \\
\text { riego? }\end{array}$ & $\begin{array}{l}\text { P:¿A basteci } \\
\text { miento su- } \\
\text { ficiente? }\end{array}$ & $\begin{array}{l}\text { P:¿Frecuencia } \\
\text { con queredibe } \\
\text { el aguaopor- } \\
\text { tunamente? }\end{array}$ \\
\hline $\mathrm{R}$ : A favor & R:No & R:Sí & R:Máságiles. & R:Sí & $\begin{array}{l}\mathrm{R}: \text { Siempreo } \\
\text { casi siempre }\end{array}$ \\
\hline V:67\% & V:62\% & V: $82 \%$ & V:75\% & V: $67 \%$ & V: $80 \%$ \\
\hline $\begin{array}{l}\text { Nivel designificancia } \\
\text { (dependendia) } \Longrightarrow \gg>\end{array}$ & Ns: 0.015 & Ns: 0.201 & Ns: 0.001 & Ns: 0.071 & Ns: 0.037 \\
\hline
\end{tabular}

$\mathrm{P}:=$ pregunta; $\mathrm{R}:=$ respuesta predominante; $\mathrm{V}:=$ proporción deusuarios.

Fuente: Elaboración propia con base en resultados de la encuesta "La modernización del campo: implicaciones agroproductivas y ambientales en el valle de Mexicali", secciones II, III y IV. 
y, adicional mente, con los que aseveran disminución detrámites para el servicio de riego. Se muestra también la dependencia de esta respuesta en la percepción, con la de los que indicaron mejoramiento en la medición y abastecimiento suficiente y oportuno del agua.

No obstante la dependencia de los factores de autogestión y uso y manejo mostrados respecto a la percepción actual, los niveles de significancia muestran la estrecha relación de respuestas y, por ende, deexplicación delos factores burocráticos.

Es importante señalar que uno de los objetivos principales de la transferencia del distrito, como es el de incrementar la eficiencia en el uso y manejo del agua, en el estudio seobtuvo queno seclasifica como factor preponderante deexplicación del cambio deposturas a favor de la transferencia.

\section{CAPACIDAD DE AUTOGESTIÓN DE RECURSOS HIDROAGRÍCOLAS}

Algunosindicadores decapacidad deautogestión seobtuvieron a partir de los siguientes aspectos: dificultades detectadas para la real ización de actividades sustantivas; complementariedad o duplicidad de funciones entre el módulo de riego y otros entes gubernamentales 0 civiles; desarrollo organizacional; nivel de inversión en obras hidroagrícolas y tramitología interna y/ o externa.

De esta manera, se obtiene que una proporción el evada (62\%) de los usuarios no considera que existan dificultades en las actividades del módulo. No obstante, poco más delatercera parteopina lo contrario, y subraya que las dificultades se refieren básicamente a la administración realizada por los directivos del módulo. ${ }^{8}$ Deestemismo subgrupo menor deusuarios, $70 \%$ indica queello genera conflictos en

\footnotetext{
${ }^{8}$ La asociación de usuarios basa su actividad y formación en los títulos deconcesión, estableciendo en el acta constitutiva de la asociación civil, la elección del consejo directivo y el consejo devigilancia. El primero lo integran: el presidente, secretarios administrativo y deactas, y el tesorero. Esteórgano ejecutivo se rige por la máxima autoridad que es la asamblea general de asociados, pero tiene facultades para nombrar al personal operativo del módulo deriego.
} 
el siguienteorden y nivel deinterrelación: a) entreusuarios y gerencia del módulo, y b) únicamente entre usuarios. Al respecto, debetenerse en consideración que un conflicto del primer tipo, incrementaría la posibilidad de desencadenar una serie de dificultades de operación hidráulica y que implicaría problemas en el servicio de riego. Sin embargo, la parte más crítica es la de los conflictos entre los usuarios receptores del servicio, por su naturaleza decompetencia por el recurso. Esto induce una potencial ruptura de la armonía de subsectores productivos (algodoneros, alfalferos, trigeros, ganaderos, etcétera) y detipos de productores correspondientes al sector social y al privado.

Delo anterior se desprende que, no obstantela mínima proporción de usuarios que identifican dificultades en las actividades inherentes al módulo deriego, éstas no son predominantes, aunqueexisten riesgos potenciales de provocar crisis de autogestión en caso de no controlar las "inconformidades". Las entrevistas a profundidad permiten discernir las consideraciones de diversos actores usuarios respecto a las dificultades quese han encontrado. ${ }^{9}$ En primer término se señalala recién adquirida experiencia, pero en franca tendenciaala estabilidad, manejable hacia adentro del módulo de riego.

Por otra parte, existen factores geográfica y administrativamente externos que afectan la actividad interna y que se consideran difíciles decontrolar mientras no seestablezca un nivel organizacional superior deautogestión; es decir, la sociedad deresponsabilidad limitada (S. de R. L.), que agruparía a los módulos de riego para contemplar la operación dered mayor de irrigación y las fuentes deagua superficial (Río Col orado) y subterráneas ( 725 pozos profundos). No obstante, en la región estudiada se manifiestan dudas sobre su funcionamiento, aspecto que se refleja en el hecho de que $52 \%$ de los usuarios niegan o dudan que la formación de la S. de R. L. apoyaría las actividades internas del módulo, y que en todo caso, provocará diferencias entre

${ }^{9}$ Para complementar el análisis del tema dela autogestión se real izaron entrevistas a profundidad a los diferentes actores vinculados con ello: funcionarios dela CNA y del gobierno del estado, autoridad directiva y operativa del módulo de riego y, finalmente, usuarios de los sectores social y privado. 
módulos de riego. Por otra parte, la proporción de usuarios que opina positivamente, prevee mejoramiento, principal mente en la eficiencia en el manejo del agua, por ende, en la operación hidráulica.

Un factor fundamental y de alta certidumbre para indicar una autogestión eficiente, es la realización de obras de infraestructura. AI respecto, $82 \%$ de los usuarios respondieron que después de la transferencia se incrementó dicha actividad. Este hecho muestra un avance significativo en caso de una autonomía real , sin embargo, aún es notoria la participación oficial a través de programas complementarios delacNA. Deuna u otra forma, la mayoría de los usuarios aceptan esto debido al beneficio queen lo general reciben, mismo que setraduce en conservación de drenes, revestimiento de canales e instalación o rehabilitación decompuertas (en eseorden defrecuencia). N o obstante, las autoridades ejecutivas y operativas del módulo observan esto como una desventaja que, en la mayoría delos casos, obstaculiza el desarrollo de una auténtica autogestión.

Cabe señalar que si bien la mayoría (82\%) de los usuarios indican que se incrementó la realización de obras en el módulo de riego, sólo $48 \%$ de ellos mencionó que se han visto beneficiados directamente, sobretodo con trabajos para mejorar la eficiencia en el uso del agua de riego. Esto último obliga a la reflexión cuando se cruza con el análisis anterior que determina como factor secundario decambio positivo de actitud de los usuarios, al mejoramiento de la eficiencia en el uso del agua. En otros términos, la pregunta queaflora es quesi bien mínimas, ¿quétan efectivas han sido las obras realizadas con ese fin?

Un factor adicional indicador del nivel y calidad deautogestión, es la tramitología. Se considera para tal efecto, que ésta se efectúa para atender las funciones básicas dela gerencia técnica del módul o deriego. En esteaspecto, $75 \%$ delos usuarios afirman que los trámites son más ágiles, y sólo $22 \%$ manifiesta que no hay cambio en los procesos en comparación con el periodo anterior a la transferencia, periodo en el que la CNA se hacía cargo de dichas funciones. Las entrevistas a profundidad con autoridades oficiales de los diferentes niveles de gobierno coinciden con la opinión de los usuarios en cuanto a la disminución de la actividad burocrática y mejoramiento en los procesos operativo-administrativos (ver cuadro 6). 
CUADRO 6. Características de la tramitología después de la transferencia.

\begin{tabular}{|l|c|}
\hline Característica & Proporción deusuarios quelo afirman \\
\hline Más ágiles & $75 \%$ \\
Menos ágiles & $3 \%$ \\
Iguales que antes & $22 \%$ \\
\hline
\end{tabular}

FUENTE: Elaboración propia con base en resultados de la encuesta “La modernización del campo: implicaciones agroproductivas y ambientales en el valle de Mexicali", sección II, Percepción sobre la transferencia del distrito de riego.

Asimismo, los usuarios queopinan negativamente, exponen quela principal forma en quese ven afectados, es en el retraso de entrega de volúmenes deagua, desfasamiento defechas desiembra y del proceso productivo en general, con implicaciones de potencial pérdida de productividad y mercado.

Uno de los temas considerado instrumento toral para lograr los objetivos fundamentales de la transferencia - la autosuficiencia económica- y paralelamente los de la autogestión, es el aspecto financiero, estructurado con base en el sistema decuotas por servicio de riego. De esta forma, se disciernen al gunos aspectos que aclaran el panorama para profundizar en la amplia gama de análisis que se derivan del tema particular.

En principio se evidencia la confusión y desconocimiento de los procedimientos, mecanismos y estrategias para el establecimiento de las cuotas. Al cuestionar a los usuarios sobre si consideran adecuado el precio quepagan por el agua, 42\% indicó quees justo; una proporción similar dijo quelo consideraba al to; proporciones menores de usuarios (8\%) indicaron desconocimiento y/ o quees bajala cuota. Ello confirma lo queseasienta con anterioridad, y secomplementa con la propuesta de precio por parte de los usuarios, misma que arrojó una amplia dispersión de valores referenciados al actual de 2.50 pesos, y que van de 1 a 4 pesos Ips/ 24h (unidades volumétricas en litros por segundo por cada 24 horas). 
Se obtuvo, adicionalmente, que $60 \%$ de los usuarios han visto reflejado el impacto del incremento en las cuotas, básicamente en aspectos de conservación y realización de obras. No obstante, existe aún una alta proporción de usuarios con incertidumbre respecto a lo que las cuotas pueden aportar en beneficio del módulo de riego en su conjunto, y en lo individual a nivel de parcela.

Delo anterior sedesprendela preocupación por el estudio profundo del tema de las cuotas. Éste representa un factor determinante para lograr una autogestión eficiente, según lo expresan en las entrevistas a profundidad las autoridades ejecutivas y operativas del módulo de riego.

MEJORAMIENTO DEL USO Y MANEJO DEL AGUA DE RIEGO: OBJETIVO CENTRAL DE LA TRANSFERENCIA Y RETO PRINCIPAL DE LA AUTOGESTIÓN DE USUARIOS

En este apartado se amplía lo que al respecto del uso y manejo del agua se ha mencionado en la percepción y elementos generales de gestión derecursos hidroagrícolas. Seresalta el tema, por considerarse un aspecto central tanto del propio proceso detransferencia, como del desarrollo de la autogestión. Así, por separado los aspectos de abastecimiento, medición, oportunidad hacia dentro del módulo por un lado, y los programas espeć́ficos oficiales, por otro, aterrizan en indicadores deavance o retroceso, estableciendo un comparativo entre las condiciones antes versus después de la transferencia del distrito de riego a los usuarios.

Se parte de una afirmación general delos usuarios, que distinguen y clasifican el uso y manejo del agua en su región. En el escenario comparativo antes mencionado, $65 \%$ de los usuarios indicaron que mejoró, 23\% considera que no hay cambio, y sólo una proporción del $10 \%$ asevera queempeoró. Es notorio el predominio delas dosterceras partes queseñal an avances en dicha actividad en un lapso de 5 añosy bajo diferentes condiciones.

Profundizar en las particularidades, permite corroborar, o contradecir en su caso, la opinión general expresada (ver cuadro 7). Así, en el aspecto demedición degastos hidráulicos servidos para riego, más de 
la mitad delos usuarios niega o desconoce queha mejorado el proceso realizado ahora por los usuarios. Esto se relaciona con el evidente desconocimiento de acciones compartidas por usuarios y organismos oficiales emprendidas para tal fin, como es el caso del Programa de Dotación Volumétrica, mismo que se maneja desde los inicios de la transferencia y del que $70 \%$ de los usuarios desconoce sus bondades, alcances y mecanismos de operación. Pese a ello, de la mínima proporción que sabe acerca del programa, se rescata lo queel usuario entiendepor contar con un sistema de medición preciso: disminución de pérdidas de volúmenes, y con ello el potencial incremento de superficies deriego.

En cuanto al abastecimiento, el problema sereduce, ya que $67 \%$ de los usuarios expresaron que sus demandas de volúmenes para riego eran atendidas satisfactoriamente, detal manera queel agua solicitada "coincidió" con la que se entregaba. Aquí, debe considerarse la deficiencia en sistema demedición. A esto, seañadeotro punto a favor referentea que $80 \%$ delos usuarios indican quesiempreo casi siempre reciben el agua oportunamente. ${ }^{10}$

Los factores intrínsecos mencionados son, a la vez, elementos para evaluar la autogestión, ya que al inicio de la transferencia se establecieron como las principales fallas, y por ello significaron un reto para los usuarios.

Otro factor catal ogado como externo, pero que igual mente induce una opinión sobrela autogestión, es el establecimiento de programas oficiales para mejorar el uso y manejo del agua de riego, y al respecto se midió la diferencia cuantitativa y cualitativa de éstos. Sobresale la afirmación que hace $35 \%$ de los usuarios indicando que después de la

${ }^{10}$ Esto se traduce en el hecho de que, actualmente, es más a tiempo la entrega de agua, en promedio cuatro de cada cinco riegos. 
CUADRO 7. Factores de cambio en el uso y manejo del agua en el contexto dela autogestión.

\begin{tabular}{|lc|cc|ll|}
\hline ¿Mejoróla medición? & \multicolumn{2}{|c|}{$\begin{array}{c}\text { ¿A bastecimiento } \\
\text { suficiente? }\end{array}$} & \multicolumn{2}{c|}{$\begin{array}{c}\text { ¿Recepción oportuna } \\
\text { del agua? }\end{array}$} \\
\hline Sí & $49 \%$ & Sí & $67 \%$ & Siempre & $43 \%$ \\
No & $38 \%$ & No & $30 \%$ & Casi siempre & $37 \%$ \\
No sabe & $13 \%$ & No sabe & $3 \%$ & $50 \%$ de las veces & $7 \%$ \\
& & & & Pocas & $13 \%$ \\
\hline
\end{tabular}

FUENTE: Elaboración propia con base en resultados de la encuesta "La modernización del campo: implicaciones agroproductivas y ambientales en el valle de M exicali", sección IV, Uso y manejo del agua de riego.

transferencia ha habido mayor cantidad deprogramas específicos; $27 \%$ -que es una proporción importante - afirma que se mantienen sin cambio entre un periodo y otro; $23 \%$ desconoce si se ha establecido este tipo de programas en su módulo de riego, y finalmente sólo $15 \%$ dijo que antes dela transferencia se establecían más programas. Dela anterior dispersión en la respuesta se deduce una fal ta deinformación en lo general, que puede confirmarse con la opinión sobre algún beneficio individual. Al respecto, en las parcelas de $88 \%$ del os usuarios no sellevó a cabo programa alguno para mejorar el uso y manejo del agua.

Por otra parte, seobtuvo queuno delos programas gubernamentales que se extendieron en alguna medida en beneficio de los usuarios y sus predios, fue el de Nivelación de Tierras, mismo que apoyó a 7\% del total de los usuarios.

De esta manera, la revisión de los aspectos intrínsecos y externos permitetener, en un escenario comparativo, una mejor visión sobrela 
importancia, pero a la vez, el cambio en materia de uso y manejo del recurso hídráulico, esto como un indicador principal deautogestión.

\section{ENFOQUESY TENDENCIASDE LA AUTOGESTIÓN DE RECURSOS HIDROAGRÍCOLAS}

Se obtuvieron opiniones generales respecto a la autogestión ubicada en los siguientes aspectos: la transferencia del distrito de riego y las acciones para mejorar el uso y manejo del agua; dificultades de la autosuficiencia económica de los módulos; ventajas y limitantes de la participación delos usuarios; desarrollo organizacional y perspectivas, así como lo referentea la problemática ambiental, punto queseaborda con profundidad en apartados posteriores.

Se han señalado algunos puntos en el que las observaciones recogidas en las entrevistas a profundidad amplían y complementan losenfoquesal respecto. El ejercicio dediscernirlos, permiteprofundizar en ellos para encontrar puntos de unión y divergencia.

De las entrevistas real izadas se rescata la diversidad deenfoques a Ios planteamientos, y se definen dos grandes convergencias generalizadas de autoridades de la CNA y del Gobierno del estado a través dela Secretaría de Fomento A gropecuario (SFA), así como delos usuarios desdesu posición directiva-ejecutivay dereceptor del servicio de riego: los cambios positivos y las dificultades económicas referenciadas a condiciones comparadas de los periodos anterior y posterior a la transferencia del distrito de riego.

Los cambios positivos versan sobrela adecuación del os productores a una nueva realidad competitiva, la mayor concientización sobre el valor económico-productivo del agua y la tendencia hacia el desarrollo de infraestructura hidroagrícola para incrementar la eficiencia del servicio de riego. En tanto que las dificultades derivan de aspectos financieros, específicamente de los esquemas tarifarios que inducen problemas esenciales parallevar a cabo una eficientegestión. Las cuotas son y serán un punto crítico en este contexto.

Algunos puntos analizados arrojan diferencias deopinión entrelos actores. Las limitantes autogestivas son observadas con diferente enfoque: las autoridades estatales anotan la falta de capacitación y 
adecuación reglamentaria; los usuarios ejecutivo-operativos indican falta derecursos financieros einfluencias negativas externas al módulo de riego que atañen a entes organizacionales superiores como la sociedad de responsabilidad limitada del distrito de riego; mientras los usuarios expresan queaún fal ta adquirir experiencia en esta nueva tarea deautogestión, sobre todo en la operación hidráulica.

El aspecto organizacional es otro punto de convergencia dondelas opiniones complementan y amplían el panorama. La formación delaS. deR. L. sevislumbra como un factor deimpul so al desarrollo individual delosmódulos deriego, a través del mejoramiento en la conservación y operación dela red mayor de riego. Sin embargo, el sector de usuarios profundiza y pone como condición para que este desarrollo organizacional se dé satisfactoriamentey con los beneficios esperados, definir con cautela al personal queparticipará en ese ente superior.

Final mente, la perspectiva a futuro es interesante distinguirla para cada uno de los actores en el proceso de autogestión. Aquí salen a relucir dos aspectos expresados: desde la perspectiva oficial y de los ejecutivo-operativos del módulo de riego, el nivel y calidad organizacional son definitivos en el desarrollo sostenido de un ente autogestor autosuficientetécnica, organizativa y económicamente, así ponen en primer plano la no politización de las estructuras establecidas para la autogestión (módulo de riego en primera instancia, y S.de R. L., en segunda). El sector de usuarios receptores del servicio, por su parte, prioriza los aspectos de concientización y de incremento permanente de infraestructura hidroagrícola en busca de mejorar el uso y manejo del agua, detal manera que los ubique en una posición más competitiva.

\section{CONCLUSIONES}

Los instrumentos básicos de administración observan limitantes esenciales, ya queno secuenta con un documento veraz queidentifique cualitativa y cuantitativamente a los integrantes de la asociación, aspecto que induce desajustes y distorsiones en las actividades normales del módulo de riego, que se transforman en ineficiencias técnico-administrativas. A dicionalmente, seobserva el mínimo grado 
de automatización y sistematización de los controles estadísticos, ya que entre $75 \%$ y $80 \%$ de los formatos son capturados manualmente y serefieren a la información sobreconservación y operación.

La percepción del usuario respecto al proceso de transferencia, en términos genéricos indica el mejoramiento y su aceptación. Sin embargo, en la medida que revisan especificidades, la percepción se torna inconsistenteen al gunos aspectos. Se identifican mejoras reales con el hecho de transferir funciones a los usuarios, la más palpable es la reducción al mínimo de los trámites burocráticos, persistiendo aún (después de cinco años) diversas restricciones financieras, legales, de capacitación técnica, administrativa y de desarrollo organizacional, factores que ponen en riesgo lo que se ha avanzado y los intereses productivos al interior del módulo de riego y con los que interactúan que, por cierto, han mostrado problemáticas similares (seabastecen de la misma forma y fuente de agua).

Por otra parte, se evidencia la participación insistente por parte de la CNA, sobre todo en cuestiones organizacionales, aspecto que se considera limitativo para los usuarios. En contraparte, en otras áreas donde se requiere amplíe su presencia como los reglamentarios, de inversiones y capacitación técnica transicional, su función se considera incompleta. Debe, entonces, analizarse la forma en que las instancias gubernamentales participan, deslindar su responsabilidad y atender adecuadamente las que le corresponde, con objeto de coadyuvar al mejoramiento de la autogestión de recursos hidroagrícolas en los módulos deriego.

Es notoria la tendencia a mejorar el uso y manejo del agua en el contexto dela autogestión, no obstantequeen el periodo queseanaliza este tema se catal oga como secundario.

El módulo deriego estudiado es representativo dela región agrícola, lo cual ofrece certidumbre para extrapolar resultados. Sin embargo, se recomienda adecuar a condiciones particulares, profundizar y ampliar a nivel de distrito de riego o realizar análisis comparativos intrarre gionales.

Por otra parte, es importante señalar queel cambio inducido por el índice de rentismo de la región, indica la prioridad de actualizar el padrón de usuarios, ello como el emento básico de planeación y 
autogestión. Por tal razón, es recomendable, para estudios futuros en esta área temática, considerar las modificaciones de su estructura. En el estudio realizado, este aspecto representó un esfuerzo adicional metodológico. ${ }^{11}$

Final mente, semenciona la importancia que este trabajo tiene en el contexto de un proceso en donde los actores sociales del campo -en este caso los usuarios hidroagrícolas- juegan un papel primordial. A esto se añaden factores como los de la ubicación geográfica de los mismos. El hecho deestar en una región fronteriza y compartir recursos transfronterizos como el agua de riego, es uno de los retos adicionales que tendrán que enfrentar los hoy autogestores de los recursos hidráulicos e infraestructura. En este sentido, este trabajo aporta experiencias de una subregión fronteriza con margen razonable de comparación y/ o extrapolación, en su caso, primeramente al mismo valle de Mexicali, por las características similares que en lo físico y socio-productivo tieneel módulo deriego número 15, y con otras zonas agrícolas fronterizas, por la similitud de procesos que se presentan.

${ }^{11}$ En el estudio realizado se obtuvo que la proporción de usuarios que dejaron de trabajar sus parcelas en el periodo de 1993 a 1997, pasó del 30\% al 70\%, proporción equivalenteal incremento del rentismo en esta zona, aunque en al gunas zonas del valle de Mexicali la proporción es mayor de $85 \%$. Esto podría representar un factor que distorsiona la percepción real del usuario sobre el proceso de transferencia, y en términos generales, su visión de autogestión. 


\section{BIBLIOGRAFÍA}

Castro, J. (1995). “Descentralization and Modernization in M exico: The Management of Water Services", N atural Resources Journal, vol. 35, Nueva York.

Comisión Nacional del Agua. Distrito deriego 014, Río Colorado (1993).

"A nexos del Título de Concesión, Usuarios del Módulo 15, margen derecha del Río Colorado, A.C.", Mexicali, B.C.

Cortez, L.A. (1996). “Viabilidad económica y financiera deinversiones para infraestructura hidroagrícola en módulos del distrito deriego 014, Río Colorado", tesis demaestría, UABC, Mexicali, B.C., México.

— (1997). “Análisis del impacto técnico-económico de la obra de nivelación de suelos agrícolas en módulos de riego del distrito 014, Río Colorado", reportefinal deinvestigación, El Colegio de la Frontera N orte, M exicali, B.C.

Dourojeanni, A . (1994). La gestión del agua y las cuencas en América Latina, Revista de la CEPAL, núm. 53, México.

Escamilla, M. y Kurtyz (1995). Social Participation in the Lerma-Santiago Basin: W ater and Social Life Project.

León, D. (1996). “Diagnóstico dela situación del distrito de riego 051costa de Hermosillo ante los cambios en la gestión del agua", Revista de El Colegio de Sonora, vol. VII, núm. 11, Hermosillo, Sonora, México.

Roemer, A . (1997). D erecho y economía: Políticas públicas del agua, Centro de Investigación y Docencia Económica-Sociedad Mexicana de Geografía y Estadística, Ed. Porrúa, México.

Whiteford, S. y Cortez, A. (1996). “Conflictos urbano-rurales sobreel agua del Río Colorado en el ámbito internacional", en: C. Vargas y Sosa (coords.), A gua: desafíos y oportunidades para el siglo XXI, Aguascalientes, Ags., México. 